Questions vives

\section{Questions Vives}

Recherches en éducation

$\mathbf{N}^{\circ} 24 \mid 2015$

Accompagnement des transitions professionnelles et dispositifs réflexifs en formation initiale et continue

\title{
Écriture de cas entre stagiaires, étudiants de pédagogie et leurs tuteurs, professeurs en exercice : un dispositif pour la professionnalisation enseignante
}

Writing cases between novice and experienced teachers (mentors): a device for the professionalization of teachers

\section{Mauricio Núñez Rojas}

\section{(2) OpenEdition}

1 Journals

\section{Édition électronique}

URL : http://journals.openedition.org/questionsvives/1818

DOI : 10.4000/questionsvives. 1818

ISSN : 1775-433X

Éditeur

Université Aix-Marseille (AMU)

\section{Édition imprimée}

Date de publication : 15 décembre 2015

ISBN : 978-2-912643-48-3

ISSN : $1635-4079$

\section{Référence électronique}

Mauricio Núñez Rojas, «Écriture de cas entre stagiaires, étudiants de pédagogie et leurs tuteurs, professeurs en exercice : un dispositif pour la professionnalisation enseignante », Questions Vives [En ligne], № 24 | 2015, mis en ligne le 24 mars 2016, consulté le 02 mai 2019. URL : http://

journals.openedition.org/questionsvives/1818; DOI : 10.4000/questionsvives.1818

Ce document a été généré automatiquement le 2 mai 2019.

\section{(c) $($ ) $\odot$ (8Y NC ND}

Questions Vives est mis à disposition selon les termes de la licence Creative Commons Attribution -

Pas d'Utilisation Commerciale - Pas de Modification 4.0 International. 


\section{Écriture de cas entre stagiaires, étudiants de pédagogie et leurs} tuteurs, professeurs en exercice : un dispositif pour la professionnalisation enseignante

Writing cases between novice and experienced teachers (mentors): a device for the professionalization of teachers

\section{Mauricio Núñez Rojas}

\section{Introduction}

1 Écrire constitue une action essentielle inhérente à notre entité. Écrire de façon récursive est un acte qui redonne une signification à notre existence. Écrire de façon réitérative au sein d'une communauté de pratique, tout en étant à l'abri des regards univoques, est une démarche de la pensée qui nous forge en tant que membres de ladite communauté.

2 L'écriture, en tant que pratique, est un élément central dans tous les processus de construction d'une professionnalité réflexive. Néanmoins, faire écrire est une demande exigeante. Cognitivement c'est un défi majeur, particulièrement aujourd'hui dans une société qui montre des signes d'une certaine « agraphie » qui n'est pas liée à un problème physique, mais à un problème culturel. En effet, selon la définition de Castro, «l'analphabétisme fonctionnel représente la situation de l'individu qui, tout en sachant lire et écrire, n'exerce pas ces acquisitions et, dans les faits, est analphabète puisqu'il n'utilise pas ce qu'il sait » (Castro, 2001, p. 29) ${ }^{1}$. Pour écrire, il faut s'arrêter, faire une pause, faire du silence, et tout cela prend du temps, un temps dont nous ne disposons plus, car il nous a été volé par les exigences de la modernité et la logique de la productivité. Chez les enseignants, cette condition est une conséquence directe de 
l'adaptation du système éducatif au modèle de production et des nouvelles exigences qui s'en dégagent, qui ne sont plus aujourd'hui d'ordre intellectuel, mais plutôt d'ordre technique. Notre postulat de base considère la professionnalisation enseignante comme le résultat d'une praxis (réflexive, consciente) (Freire, 2002), où la construction du sujet devrait prévaloir contre l'efficience, raison pour laquelle nous demandons du temps. « Du temps, du temps, donnez-nous du temps", réclame une poète québécoise, tout en cherchant à rescinder le dommage induit par le temps perdu, l'oubli du temps (Laforce, 2000), ce temps dont nous avons besoin pour nous retrouver et pour faire de la pratique enseignante une praxis.

Par cet article, et à partir d'une expérience de recherche en cours, nous voudrions proposer et discuter l'idée d'un dispositif de professionnalisation permettant une prise de conscience et une double transition : celle de l'entrée dans le métier d'enseignement chez les étudiants, futurs professeurs et celle du passage du rôle d'enseignant vers celui de tuteur de stage chez ceux qui sont plus expérimentés. Un tel dispositif implique donc une rencontre intergénérationnelle des sujets en formation et les amène à faire un bout de chemin ensemble, pour vivre un parcours de professionnalisation qui prendra forme dans et par l'écrit.

\section{Contexte de la recherche}

\subsection{Les stages professionnels au Chili}

Dans le monde des institutions de formation des maîtres, il n'est pas facile de trouver une juste équivalence entre les personnes qui font le travail de formation et d'accompagnement des stagiaires dans les écoles et les dénominations utilisées par leurs propres institutions. C'est ainsi que nous pouvons trouver des fonctions semblables qui sont nommées différemment, telles que celles de tuteur, mentor, enseignant associé, responsable de formation pratique, guide de stage, etc. Dans ce texte, nous parlerons de tuteur, dénomination qui sera comprise dorénavant comme le sujet qui, en collaboration avec les formateurs universitaires, mais tout en demeurant praticien à l'école, appuie la formation des étudiants de pédagogie dans le volet pratique du métier. Il s'agit d'un enseignant ou d'une enseignante devant être reconnu par son expérience pratique. Cependant, ce n'est pas toujours le cas, au moins dans le contexte des écoles chiliennes et pas davantage dans le reste du monde. Les enseignants considérés comme experts universitaires ne sont pas aussi nombreux que les étudiants en formation des maîtres qui arrivent dans les écoles afin de faire leur stage. C'est ainsi que les professeurs qui deviennent tuteurs de stages sur le terrain n'ont pas toujours suffisamment d'expérience ou bien sont engagés par la direction de leur établissement, en raison des accords institutionnels signés entre l'école et l'université. Parfois, ils expriment des divergences avec les approches prises par les formateurs universitaires, allant même jusqu'à la confrontation de points de vue sur l'enseignement, sur la gestion de la classe, ou bien ils manifestent clairement la difficulté d'accomplir cette fonction qui devient à leurs yeux une exigence qui s'ajoute à leurs tâches quotidiennes. 


\subsection{La problématique des articulations théorie-pratique en formation}

5 Dans le cadre de la formation initiale, les stages en milieu scolaire ont, au Chili, une composante relationnelle assez complexe par rapport à la place et au rôle que possèdent les enseignants tuteurs dans la formation des étudiants de pédagogie stagiaires. La pratique du mentorat ne constitue pas toujours un véritable accompagnement et les relations de collaboration se font à des degrés divers. À partir de notre travail en formation initiale des enseignants à l'Université du Chili ${ }^{2}$, nous avons constaté qu'il y a des lacunes et des incohérences entre l'expérience des stages scolaires et la pratique professionnelle quotidienne (Schön, 1992). L'Université attend de ses étudiants le développement d'un regard critique sur le système scolaire. Le système scolaire pose à son tour ses propres requêtes aux enseignants souvent associées aux politiques publiques, et qui n'ont pas toujours l'appui des facultés d'éducation (Shulman, 2005). C'est ainsi que ces deux regards se confrontent dans le milieu de la pratique. Mais, le problème ancré dans le rapport établi entre le monde de la pratique et celui de l'université est beaucoup plus complexe. Dans ce dernier, il y a des personnes qui argumentent encore qu'en tant que formateurs, nous ne pouvons pas laisser les étudiants assimiler la façon de raisonner des praticiens car ils sont, pour la plupart d'entre eux, des survivants, adaptés au système alors que celui-ci est partout en crise. D'autre part, nous savons que les enseignants, ayant accumulé des années de pratique, ont fait face à de nombreuses situations dont nous pourrions nous inspirer. Mais, pour cela, la publication devient une condition, soit par des rapports de recherche ou par des récits de pratique par exemple. Cependant, le monde académique a eu tendance à ignorer la voix des enseignants (Elbaz, 1991; Nieto, 2005). Même les étudiants de pédagogie en stage ont tendance à ignorer la valeur de l'expérience de leurs enseignants-tuteurs. Une des conséquences de cette réalité est de perdre la richesse de la connaissance pratique commune, qui exprime la façon de gérer les problèmes, les besoins des praticiens et les exigences de la réalité scolaire. Cette richesse des connaissances accumulées et explicitées de la communauté scolaire pourrait servir à la fois de base pour le jugement professionnel et pour l'action, ainsi que pour former les opinions pratiques des futurs professeurs afin qu'ils arrivent à penser en tant qu'enseignants (Kleinfeld, 1992). Néanmoins, nous croyons que la conséquence la plus importante et troublante est celle de voir se rompre les liens vitaux entre les acteurs de l'éducation, ceux que devraient soutenir le dialogue théorie-pratique et la construction de la professionnalité tout au long de la vie.

6 Mais, afin d'être juste concernant ce portrait de ceux qui devraient être nos plus proches collaborateurs en formation des maîtres, il convient de signaler que, dans le cas de la profession enseignante au Chili, il y a d'énormes difficultés et contraintes par rapport aux conditions contractuelles et de travail des praticiens ${ }^{3}$ : flexibilité dans les contrats qui n'offraient pas de sécurité aux postes de travail, surcharge dans les responsabilités d'enseignement de l'ordre de 44 heures de cours par semaine, une évaluation et une régulation permanente de l'enseignement par l'application de tests standardisés pour les élèves et la classification des écoles selon ces résultats. Bref, des écoles livrées au marché économique et cela étant, aucune possibilité de travail réflexif pour les enseignants. Alors, même si nous ne pouvons pas nier qu'il y a de bonnes expériences avec quelques tuteurs, nous constatons que, en général, avec ces conditions, il existe rarement une 
disposition initiale favorable à une collaboration en formation des maîtres. Le contexte est par trop contraire.

7 L'Université du Chili offre un programme de formation initiale consécutivement à l'acquisition d'une licence disciplinaire de base (ou baccalauréat). La conséquence directe de ce modèle est de recevoir en début de formation initiale des étudiants plus âgés dont la maturité est tout autant intellectuelle qu'émotionnelle, même s'ils subissent des difficultés dans leur stage, ils sont capables d'en sortir « édifiés » et ayant déjà aqcuis une certaine expérience de vie. Le problème de cette modalité est situé dans une sorte d'arrogance manifeste de plusieurs étudiants par rapport au travail des praticiens. Parfois, ils parlent d'erreurs dans les contenus disciplinaires chez leurs tuteurs, parfois d'attitudes de contrôle extrême face à une classe qui est stigmatisée. Cependant, ils les accusent très rarement de perte de contrôle.

8 Par ailleurs, la distance établie entre les stagiaires et leurs tuteurs est le résultat de la méfiance et de l'abandon, ainsi que d'un manque de reconnaissance mutuelle qui s'est installé depuis des années, d'abord entre les professeurs des écoles et les enseignants universitaires et par extension avec les stagiaires eux-mêmes.

9 Deux problèmes apparaissent : d'une part, une forme d'incommunicabilité entre stagiaire et tuteur sur les plans de la valorisation et du partage de l'expérience; d'autre part, le manque de développement d'un regard professionnel commun.

10 C'est dans ce contexte que nous avons conçu et mis en place une recherche qui permettrait de réunir tuteurs et stagiaires dans une expérience réflexive commune. Nous proposons donc un dispositif de recherche et de formation qui s'inscrit dans une démarche collaborative de professionnalisation, située dans l'espace même de l'école, avec un volet de recherche et un volet de formation (Desgagné et al, 2001). En même temps, nous souhaitons repérer dans quelle mesure ce dispositif peut devenir un modèle pour la formation des enseignants, envisagée comme un processus permanent de développement professionnel (Wittorski, 2007).

\section{Cadre théorique}

\subsection{L'expérience de la professionnalisation : un processus complexe}

11 Le concept de professionnalisation est au coeur de nos préoccupations. Cependant, comprise jusqu'à ce jour comme un itinéraire linéaire, la professionnalisation a eu tendance à séparer processus de formation initiale et processus de formation continue, ce qui en favorise la scission ainsi que la dichotomie entre la théorie et la pratique, puis la méfiance entre les acteurs de terrain et les enseignants des universités en charge de la formation initiale.

12 Si nous partons de la métaphore de l'injection, dans Connelly et Clandinin (1994), nous pourrions accorder pour la formation des enseignants une certaine logique linéaire. Mais, si nous l'abordons avec une autre image proposée par les mêmes auteurs, c'est-à-dire celle de la reconstruction, le continuum est remplacé par une certaine récursivité, une dynamique en spirale dont le départ n'est pas signé par des connaissances à acquérir, mais par des expériences à construire. Lors de l'entrée en récursivité, la biographie professionnelle est un levier intéressant. Ici, la notion de dialogisme bakhtinien (Bakhtine, 1995), par l'effet de l'autoconfrontation qu'elle suggère (Clot \& Kostulski, 
2011), vient en appui d'une conceptualisation de la «dynamique spirale» dont nous venons de parler, une dynamique qui est d'abord personnelle et aussi collective.

Certainement, la formation initiale et la formation continue des enseignants ont leurs propres logiques: l'une, celle de l'installation des bases théoriques et de l'induction professionnelle, l'autre, celle de l'enrichissement des bases de connaissances autant disciplinaires que professionnelles, en lien avec la pratique. Cependant, ces logiques sont à la fois, porteuses de certaines particularités qui pourraient enrichir la professionnalité de l'ensemble des acteurs, celle des novices ainsi que celles des plus expérimentés tout en développant la coopération mutuelle et le dialogue réflexif intergénérationnel.

Pour nous, la professionnalisation est axée sur la réflexivité (Schön, 1992) et nous comprendrons cette dernière comme étant un acte cognitif, construit sur le principe de la récurrence. Ce principe nous permet de revenir sur le vécu ainsi que sur la compréhension que nous en bâtissons par l'utilisation de l'exercice métacognitif, exprimé lors des rencontres des pairs, en communauté. Nous réfléchissons d'abord sur des événements, puis sur nos propres réflexions par rapport à ces vécus que nous transposons dans nos écrits. Mais nous travaillons aussi sur une réflexivité croisée, par l'effet de la confrontation de notre propre pensée avec les regards des pairs dans une communauté de pratique réflexive ${ }^{4}$. Ici, nous parlons de pratique dans un double sens: une pratique enseignante proprement dite, et une pratique réflexive posée sur la première, se traduisant par un exercice de maïeutique, c'est-à-dire l'art de faire éclore les grandes pensées, d'accoutumer les esprits par l'exercice à chercher et à connaître leurs facultés pour les tourner vers un but noble et utile. Dans cet exercice, la présence des étudiants stagiaires et des professeurs expérimentés dans un même espace dialogique et réflexif, se révèle comme étant promoteur d'une compréhension commune sur un espace partagé (l'école, les classes, les élèves), délivrant à chacun une expérience de professionnalisation. Cette expérience est nourrie par la communauté mais est également porteuse de particularités, propres à chaque membre, qu'il soit novice ou expérimenté.

\subsection{L'importance d'une ouverture au dialogue intergénérationnel}

15 Le dialogisme bakhtinien (Bakthine, 1995) nous parle d'une plurivocalité, constitutive de notre identité. Cette plurivocalité nous est naturelle et intrinsèque en tant que sujet d'histoires. Elle est l'expression de plusieurs voix qui viennent du passé, aussi lointain que proche, des rencontres, des lectures, des expériences qui dialoguent entre elles et s'expriment en chacun de nous, tout en révélant qui nous sommes au présent (cf. Connelly \& Clandinin, 1994). Cette constitution est pourtant aussi celle du regard que nous posons sur le monde, un regard qui relève à la fois du subjectif et de l'intersubjectif.

Cependant, une telle plurivocalité pourrait bien être activée et enrichie de façon tout à fait intentionnelle. Le dialogue intergénérationnel devient alors une manière de plonger dans une plurivocalité explicite et voulue, orientée vers la rencontre des postures, des regards, des façons d'être dans le monde et tout à fait différents en rapport avec celui-ci.

À l'école, les enseignants-stagiaires sont porteurs d'un regard nouveau, d'une fraîcheur, d'une capacité encore vive de s'émerveiller face au quotidien, de l'envie même d'avoir un impact sur la vie des jeunes. De leur côté, les plus expérimentés sont porteurs d'une sagesse de la pratique (Shulman, 1992) qui donne de la mesure, nourrit la perspective du champ d'action et permet d'agir et de poser des gestes et des jugements professionnels (Kleinfeld, 1992). La pratique peut donc permettre un enrichissement: des centaines 
d'histoires qu'ils sont capables de transformer en expériences professionnelles, puis en savoirs pratiques, à condition de franchir le seuil de l'explicitation et de la prise de conscience. Cependant, ces histoires restent là, en attente, prêtes à être dévoilées et transformées en expérience.

En ce qui concerne la dichotomie entre formation initiale et formation continue, des chemins divers ont été proposés afin de la surmonter. La figure du réseau de tuteurs (les enseignants des enseignants) en est la plus habituelle comme moyen de rapprocher les futurs professeurs des plus expérimentés. Cependant, il y a d'autres possibilités qui aident à ce rapprochement entre les perceptions des vécus. Les innovations apportées au curriculum en font partie, comme l'exprime bien Errol Miller (2002) pour ce qui est du cas de L'Association des États de la Caraïbe. Dans cette dernière, il y a des éléments communs qui sont proposés pour les programmes de formation initiale avec les méthodologies pour la formation continue, le tout dans le contexte d'une nouvelle réforme du curriculum national. Cela offrirait apparemment un terrain d'entente à partir duquel, il serait possible d'établir un dialogue fructueux entre les enseignants à différents niveaux de développement professionnel. En ce sens, quelques expériences très focalisées ont été proposées en milieu anglophone (en Australie, aux États-Unis et en Angleterre) où l'ensemble des sujets engagés en formation initiale et continue, ont constitué de vraies communautés de développement professionnel (Núñez, Arévalo \& Ávalos, 2012).

\section{Méthodologie}

\section{1. Écrire en communauté professionnelle}

Nous avons choisi par ce projet, de mettre à l'épreuve une innovation du curriculum permettant de rassembler des stagiaires avec des enseignants expérimentés (leurs tuteurs) dans un même espace (une communauté) et une même situation réflexive (un atelier d'écriture de cas réels), à caractère situé, menée en milieu scolaire. Le but de ce dispositif est de nourrir une construction distinctive chez les enseignants, comprise comme une identité «en devenir» (Malet, 1998), c'est à dire, une unité qui grandit, s'édifie telle l'expression d'un processus de professionnalisation de l'ensemble des membres, et qui est animée à partir de la réflexion autour de cas d'élèves qui sont communs au groupe. Les cas sont produits par écrit et retiennent l'intérêt des stagiaires et des professeurs plus expérimentés lorsqu'il s'agit de cas réels d'élèves qui sont les leurs.

Pourquoi réfléchir autour de cas d'élèves? La réflexion n'apparait pas spontanément, nous dit Vaillant; elle est provoquée, suscitée (2007, p. 210), et nous pensons que l'écriture des histoires d'élèves peut ouvrir tout un champ d'incidents qui nous concerne directement en tant qu'enseignants.

21 Dans cette pratique d'écriture, les textes produits sont soumis à l'arbitrage des pairs qui réalisent à leur tour des commentaires également écrits, exploités par la suite pour l'échange oral. L'espace réflexif proposé par le dispositif devient pour les uns (les stagiaires) un espace de formation et d'apprentissage d'une sagesse de la pratique, un espace qui permet de mettre en discussion plusieurs concepts théoriques qui sont appris à l'université, un espace pour reconnaitre la valeur de l'expérience enseignante. Pour les autres (les professeurs expérimentés), cet espace permet l'explicitation de savoirs pratiques, la conceptualisation de la pratique et la construction de l'expérience. 
Néanmoins, les apprentissages nommés ici sont croisés, superposés et finalement, si nombre d'entre eux peuvent être identifiés pour la formation initiale ou continue, tous semblent contribuer à la professionnalisation.

Mais, que comprenons-nous par une professionnalisation favorisée par l'écriture? Tout d'abord, il faut savoir que nous sommes ici face à un parcours de construction de l'expérience enseignante, conduisant vers l'acquisition de la professionnalité, c'est-à-dire vers l'acquisition d'une qualité professionnelle chez le professeur. Un tel parcours est le résultat de la prise de conscience développée chez l'enseignant (discursive, énonciative et même épistémique $)^{5}$ qui est issue, à son tour, d'une pratique scripturale à la fois dialogique, récursive et convergente.

Il faut dire sur ce point que chacun en est à un moment particulier dans son processus de professionnalisation et que tous n'arrivent pas en même temps à développer leur professionnalité sur le même parcours.

D'autre part, la qualité professionnelle devrait être comprise dans la notion de l'éthos, c'est-à- dire des caractères identitaires d'une communauté professionnelle. Ainsi, la professionnalité chez les enseignants devrait être en mesure d'articuler les trois dimensions suivantes : identitaire, éthique et réflexif (Desgagné, 2005).

Dans l'articulation de ces trois dimensions, ce qui rapproche de l'idée de développement de la professionnalité chez les enseignants est, pour nous, le développement de la conscience professionnelle, conscience qui s'éveille de toute évidence à travers l'écriture. Nous pourrions donc penser, à partir de maintenant, la professionnalisation comme un processus permanent de prise de conscience, une vraie transition par les divers niveaux de conscience professionnelle, un processus proche de la notion de Freire (2002), et donc de celle de libération et de transformation. En ce sens, la rencontre intergénérationnelle joue un rôle de potentialisation du développement de la conscience professionnelle, autant pour les stagiaires que pour les professeurs expérimentés.

\subsection{Bâtir des connaissances}

Le travail de formalisation de la pensée dans n'importe quel environnement professionnel peut être propice à la construction d'une base de connaissances. Nous voulions donc proposer aux enseignants un exercice de formalisation de leurs connaissances pratiques par le biais de l'exercice de l'écriture récursive et en communauté (Calderhead, 1996; Shulman, 1992).

Le projet a été mené in situ, dans un lycée public secondaire où des rencontres bimensuelles se sont déroulées pendant un an et demi, période équivalente à la durée du programme de formation consécutive des stagiaires. ${ }^{6}$ Le groupe était constitué par six stagiaires avec leurs six tuteurs de pratique (des enseignantes expérimentées du lycée) et deux professeurs universitaires chargés de l'animation de l'atelier. Il s'agissait d'un atelier d'écriture de cas. Dans cette communauté de pratique, les stagiaires sont considérés comme ayant, de plein droit, un statut similaire à celui de leurs tuteurs. L'équipe est engagée dans un exercice d'écriture et de commentaires. Puis, chaque récit qui a été produit, a été discuté en séances de rencontres en vis-à-vis. Cela permet que les cas soient systématisés à travers de multiples regards, tout en permettant la libération de la perspective individuelle unique de l'auteur. Nous parlons ici de la co-construction du regard sur un problème spécifique (Desgagné, 1997). Alors, la discussion menée en groupe 
conduit à des questionnements sur les cas : en rapport avec le texte («Qu'est ce que cela veut dire dans l'écrit, l'expression ?... »), le contexte ("Comment ils ont réagi ?...»), ainsi qu'à l'éthique professionnelle qui s'en dégage («Et quelle est ta posture à ce sujet ?...»). C'est la même communauté qui demande la réécriture du récit autant de fois qu'il est nécessaire, jusqu'à son accomplissement. Les récits de pratique produits ici ne rendent pas compte de pratiques idéales ni de problèmes déjà résolus, mais de situations uniques, singulières qui ouvrent la réflexion sur le sens des actes et des actes de paroles, susceptibles d'être communiqués, partagés et pensés.

Le nombre total d'histoires, y compris leurs versions: premières, intermédiaires et dernières a atteint un nombre approximatif de 40 récits (certains enseignants ayant écrit jusqu'à quatre versions). Le recueil des récits ainsi que des verbatim transcrits après chaque séance ont été travaillés à l'aide de Nvivo7, logiciel qui permet de cerner des catégories d'analyse découlant des textes eux-mêmes.

En résumé, toute la démarche d'explicitation de la pensée des enseignants est passée par l'écriture et l'analyse de cas d'élèves qui étaient les leurs. Un premier niveau d'explicitation était produit lors de l'écriture d'une première version des cas. Dans ce cas, c'était la perspective individuelle qui s'exprimait. Ensuite, une fois les textes donnés aux collègues et leurs commentaires produits, un premier niveau d'analyse s'exprimait lors des rencontres collectives de discussion. L'herméneutique collective, cet art d'interprétation des textes, prenait sa place et, en même temps un deuxième niveau d'explicitation était perceptible dans le feu de l'interaction orale. Chaque fois les textes et les cas étaient réinterprétés et soumis à la réécriture d'une nouvelle version. Les narrations ont été ainsi retravaillées de manière récursive jusqu'à en arriver à l'entendement commun. Pourtant, le résultat n'est pas la somme des regards isolés mais plutôt une toute nouvelle construction qui passe par la communauté tout en appartenant toujours à son auteur original. Une perspective individuelle était élargie par le biais d'une action collective de création.

Ce projet se situe clairement dans le domaine de la formation des enseignants, et plus particulièrement dans celui de la professionnalisation. Nous avons déclaré que nous envisagions celle-ci comme un processus qui comprend la formation initiale et continue en dialogue, où il $\mathrm{y}$ a des transitions et des transformations identitaires à vivre par l'ensemble des membres engagés dans ce projet. Celles-ci sont favorisées par la confrontation du vécu et des théories qui sont apportées tant pour les stagiares que pour les professeurs plus expérimentés. Ce projet a eu l'intention de rompre avec la dichotomie dominante qui a gardé les deux domaines de formation éloignés, isolés l'un de l'autre (Lieberman \& Wood, 2006; Torres, 2002). À cet égard, il n'est plus question d'articulation, mais de convergence de ces deux processus formatifs, avec leurs particularités respectives, dans une notion plus proche de celle d'un apprentissage permanent situé dans des communautés de pratique.

\section{Résultats préliminaires}

31 Dans cette section, nous présentons quelques extraits des récits produits par les enseignants engagés dans le projet, tout en décrivant l'énonciation de certaines catégories d'interprétations qui expliquent les différences entre les points de vue des stagiaires et ceux des professeurs plus expérimentés, ainsi que les transformations dont 
chacun a pu être l'objet. Ces dernières sont comprises comme le résultat des prises de conscience professionnelles, lesquelles sont repérées dans les récits.

\subsection{Le regard sur les élèves}

"Je le regarde avec un regard de professionnel expérimenté et puis je me demande : combien du temps durera-t-il dans le système? Il vient de redoubler donc,... il a connaissance de la culture scolaire. Il sait que les menaces ne sont pas plus que cela, que l'interdit aujourd'hui est à oublier demain. Il met au défi l'autorité. Il a l'acceptation de ses camarades. Il est protégé par eux, respecté par eux. Il est toujours assis au fond de la classe avec d'autres redoublants comme lui. Ils sont plus âgés, ils le prennent de haut, prennent du recul. Ils se sentent supérieurs. " [Enseignante-tuteur].

"Cependant, je ressens encore cette distance, l'impossibilité de savoir qui il est vraiment. Que pense-t-il, à quoi s'attend-il de cet espace et de ce qui nous attend? Qui est-il vraiment? Est-il le tyran du cours, le copain qui, avec une certaine brutalité, montre juste son affection et le besoin qu'il a de cette dernière? " [Enseignante stagiaire]

On repère une particularité sur la manière dont les enseignants expérimentés et stagiaires posent le regard sur leurs élèves. Le premier extrait est d'une enseignante expérimentée qui a 30 ans de pratique : «Je le regarde avec un regard de... » Nous pouvons dire que l'enseignante expérimentée porte dans son regard un composant biographique. Cette enseignante a vu plusieurs cas de redoublants pendant ses années de pratique. Elle est capable d'identifier rapidement leur profil et elle sait apparemment comment agir.

Le deuxième extrait est celui d'un récit écrit par une stagiaire. Dans son regard, à cause du manque d'expérience, il y a une composante plutôt circonstancielle.

Cependant, nous pourrions aussi interpréter autrement les deux regards et penser que celui de l'enseignante expérimentée est chargé de préjugés tandis que celui de la stagiaire est plus ouvert. Quoi qu'il en soit, les deux regards dialoguent et se nourrissent mutuellement.

Un autre récit exprime la volonté d'une enseignante de mieux comprendre l'élève qu'elle situe au centre de son récit :

"Lorsque je décide d'écrire sur lui, c'est avec l'envie de voir à travers sa visière, son "capot", l'impact du redoublement dans les écoles. J'ai l'impression que les étudiants comme Christian sont plus découragés, plus marginaux (que les autres) avec tous les mécanismes de défense activés et une faible estime de soi. Ils sont aussi, la plupart du temps, les générateurs des problèmes parce qu'ils se sentent stigmatisés " [Enseignante- tuteur].

L'enseignante cherche à comprendre l'expérience du redoublement de classe, mais elle le fait tout en partant du vécu d'un élève qui est le sien. Loin de vouloir amener une étude sociologique, elle développe un regard qui ressort d'une approche phénoménologique dont la structure se base sur l'analyse directe de l'expérience vécue par le sujet qui rend compte de cette expérience dans un rapport écrit. Il s'agit aussi d'une approche herméneutique puisque le vécu de l'élève devient pour elle une construction subjective de l'expérience du redoublement.

\subsection{Sentiments et émotions}

"Je suis excellente professeure. Je fais mon travail, j'accomplis ceci, cela, mais je mets de côté... ce qui est mortifiant pour moi aujourd'hui, maintenant. J'ai perdu un élève... Je vais en perdre un autre et je vais continuer à en perdre. Et que dire de moi? " [Enseignante tuteur]. 
motion fait partie du vécu de l'enseignant, et nous avons besoins de prendre conscience de cela. L'émotion nous mobilise et peut même être à l'origine de nos prises de décisions. Dans cet extrait, nous pouvons voir s'exprimer une sensation que nous pourrions interpréter d'abord comme négative. Cependant, la mortification est vue aussi comme une expression positive lorsque l'enseignante entre dans un questionnement à caractère éthique. «Et que dire de moi ? " En d'autres mots, qu'est-ce que je ferais face à tout cela? Tout en produisant l'émergence chez-elle d'une nouvelle disposition pédagogique envers ses élèves en difficulté.

Il convient de préciser ici que le dispositif d'écriture permet l'expression du vécu subjectif, tandis que l'échange avec les pairs ouvre la dimension de l'intersubjectif. La réflexivité qui est forgée sur l'expresion de l'émotion favorise le questionnement sur l'éthique ainsi que l'émergence de nouvelles dispositions, produisant par là-même des transformations à divers degrés chez les enseignants expérimentés. En d'autres termes, il se produit des transitions dans leurs dispositions initiales, lesquelles sont habituellement caractérisées par les préjugés, pour arriver à mieux connaître et mieux comprendre leurs élèves, une fois dépassés lesdits préjugés. Lorsque cela s'est produit, les enseignants stagiaires ont exprimé qu'un des plus grands enseignements qu'ils ont retenus, a été celui de concevoir la professionnalisation, comprise en tant que reconstruction du sujet, comme étant un engagement de toute la vie. Ils ont reçu le témoignage de leurs tuteurs.

\subsection{Des dispositions pédagogiques}

"Quand je donne des cours dans d'autres classes, parfois je vois la mienne par la fenêtre, et je me sens heureuse de savoir que Camille est heureuse et qu'elle rit avec ses camarades." [Enseignante- tuteur]

«Ce que je dis: "Toi, étudiant Christian, avec toutes ces caractéristiques qui t'appartiennent,... Oui, je tiens à toi" Et depuis, j'ai écrit ceci, j'ai commencé à penser. J'ai beaucoup pensé à comment devrait être mon plan B. » [Enseignante- tuteur]

"Ce garçon a des problèmes et j'en suis conscient. Comme je suis conscient, je vais voir comment traiter ce garçon qui a beaucoup plus un pied dehors que dedans le lycée." [Enseignant- tuteur]

Les nouvelles dispositions qui sont le résultat de la prise de conscience (des actes, des paroles, des découvertes par rapport à soi-même) sont issues de la mise en relation entre le vécu, les réflexions posées sur celui-ci et les questions sur l'éthique qui s'en dégagent. Les enseignants se questionnent à propos des changements qu'ils doivent opérer afin de répondre aux nouvelles compréhensions qu'ils ont développées au sujet de leurs élèves ainsi que leur propre rôle de formateur. Les transitions vers ces nouvelles compréhensions de soi-même, individuelles et collectives et les suivis des nouvelles dispositions pédagogiques deviennent un but de l'action formatrice dans ce projet.

\subsection{Formes plus complexes de conscience professionnelle}

"Je crois que, en tant qu'enseignants, nous sommes plus habitués à traiter avec des classes qu'avec des individus. Puis, vous finissez par ne pas savoir comment traiter les individus particuliers. Il est supposé que tous les êtres humains sont spéciaux et différents et l'on devrait être en mesure de savoir comment faire face à cette diversité... Vous dites: "Comment voir la différence quand vous avez vingt groupes différents, avec 38 personnes dans chaque classe?" C'est difficile, mais c'est en rapport au fait qu'on ne voit que des groupes et non des individus. » [Enseignant -tuteur] 
Il résulte, dans cet extrait, l'évidence que l'exercice d'écriture génère une conscience accrue de la façon dont l'enseignant comprend son action. Pour un lecteur externe, une telle réflexion pourrait paraitre du sens commun. Ce n'est pas le cas pour les praticiens qui plongent dans le champ d'action. La pratique d'écriture favorise la complexification du regard ainsi que de la pensée. Plus loin, l'enseignant dira "et maintenant, tout en écrivant, je me rends compte... " Se rendre compte, c'est être capable de reconnaître l'habitus que signe l'action professionnelle elle-même, ce qui ouvre les portes à de nouvelles possibilités et à de nouvelles dispositions d'action. L'éveil de la conscience transforme l'action. La question qui reste à résoudre est donc :

Comment parvenir à ce que la formation initiale et le développement professionnel continu ne reste pas de la simple rhétorique mais aient vraiment une incidence sur ce qui se passe dans la salle de classe et sur ce que les élèves apprennent? (Vaillant, 2007, p. 209).

Nous croyons pouvoir répondre à cette question par l'exposé d'un extrait du récit produit par un des nos enseignants stagiaires. Il s'agit d'un produit final qui exprime une problématique par rapport à l'enseignement de la philosophie.

L'auteur de cette histoire (à qui nous empruntons deux brefs extraits de son récit), relève une didactique qu'il essaie de mettre en œuvre dans le cadre de son stage final. Il s'agit d'une didactique du dialogue qui met l'accent sur l'expression des conceptions, des représentations initiales des élèves, ainsi que de l'émergence de la question au centre de la réflexion. Au cours de l'histoire, nous nous déplaçons, en tant que lecteur-spectateur, vers le sens de la relation qu'établit le praticien avec un de ses élèves, Franco, celui qui a eu une plus grande résistance au style d'enseignement qui lui avait été proposé.

Franco se révèle en effet comme étant un élève « difficile », qui rejette toute proposition à apprendre d'une nouvelle manière, différente de celle à laquelle il était habitué, ainsi que ses camarades de classe. Un élève duquel "on ne pourrait pas tirer grand-chose", lorsqu'il se refuse systématiquement à participer au cours tout en restant en silence au fond de la classe. Leurs barrières, installées si fortement protégeaient leur espace auquel l'enseignant ne pouvait pas accéder. Cependant, la relation change progressivement et l'élève commence à sortir du déni de soi. Les rencontres, les conversations avec l'enseignant, même brèves, commencent à être de plus en plus habituelles.

"J'ai eu quelques rencontres avec Franco, des conversations dans les couloirs, et même certaines interventions en classe, et qui n'ont cependant, après cela, pas empêché de me surprendre. La première étant à la fin d'une séance dont le thème était la mémoire; je quitte la salle de classe avec une sensation d'être battu, même vaincu. Je m'étais proposé de réorienter le sens de mes cours vers une décentralisation de mon rôle d'enseignant qui était devenu jusque-là trop leader, et donner dorénavant un plus grand espace à l'expression des étudiants, à leur parole. Mais aucune activité prévue n'avait captivé les élèves en dépit de mes tentatives répétées. Tout en quittant les limites étroites de la salle, laissant derrière moi mes efforts, découragé, je suis rejoint par Franco et sa voix qui frappe mon épaule: "Professeur, qu'est-ce qui ne va pas avec l'esprit, demande-t-il inquiet, j'avais onze ans avant de ne plus me souvenir de rien". Je lui réponds: "Que pensez-vous qu'il se passe?" Il continue comme en reculant, "Rien de spécial, c'est juste que je suis resté avec le doute". Je lui dis que se rappeler est important pour savoir quelle sorte de choses il aimait faire dans le passé, pour être en mesure de les répéter, comme jouer au basket dans son cas, "Imaginezvous si vous aviez oublié que vous aimiez jouer au basket, dis-je, vous vous promèneriez avec le sentiment que vous avez besoin de quelque chose et vous ne pourriez pas savoir ce que c'est, ce serait terrible... Et cela contribue également à ne pas répéter les erreurs déjà commises dans le passé". J'ai l'impression que la réponse le laisse conforme. Tout en marchant rapidement, il s'éloigne devant moi avec ses camarades, en lançant le ballon de 
basket entre eux. Je m'approche des escaliers sans être capable de deviner la cause de son intérêt soudain, lorsque je me rends compte que je souris timidement. [...]» même de façon positive. L'imprévu provoque un changement dans la relation elle-même qui devient progressivement plus ouverte, jusqu'à offrir à l'autre le sentir, la pensée, l'espérance et un sentiment de confiance qui n'avait jusque-là pas été expérimenté.

«Un autre jour, dans l'un des derniers cours que j'ai donné avant le début des manifestations des étudiants, et dont le thème était l'apprentissage, Franco m'a surpris de nouveau avec une opinion dont je retiens encore le ton. Nous nous demandions quand réalisons-nous que nous avions appris quelque chose. Victor, un des gars qui participe le plus en classe, parlait avec aisance: "Je pense que quelqu'un réalise qu'il a appris quelque chose quand il peut appliquer sa nouvelle connaissance à d'autres situations" dit-il sans hésiter. J'ai choisi des mots et je les ai écrits sur le tableau, ils étaient très proches d'une bonne définition de l'apprentissage. Mais il manquait quelque chose. Puis, je souligne à nouveau la question et cette fois, le silence s'abat sur la salle pendant un moment, jusqu'à ce que je vois planer dans l'air au fond de la salle, la main de Franco et je lui donne la parole: "Je pense que vous savez quand vous avez appris quelque chose quand vous êtes capable de l'exprimer dans vos propres mots" dit-il, et cette fois, je suis resté silencieux pendant une longue seconde, à peine capable de continuer. Le but de cette séance était que les élèves réussissent à affirmer ce qu'ils croyaient être l'apprentissage, par rapport à ce déjà vu sur le langage, et Franco n'aurait pas pu trouver une expression plus juste que celle-là. Je ne me souviens pas très bien comment il a terminé ce cours, mais il suffit de savoir que la séance suivante, lorsque nous avions à voir la question de l'intelligence, celle-ci a commencé avec ses mots. [... ]»

Vers la fin de l'histoire, Franco n'était pas juste un autre nom d'une longue liste de classe. La relation didactique a été installée : l'enseignant, le contenu, l'étudiant. Franco était allé de l'autre côté des barrières qu'il avait installées initialement et le professeur qui était au courant de ce changement, a pu apprécier chaque expression de cette ouverture. Certes, la relation avec le contenu a été aussi médiatisée par un style pédagogique de quelqu'un qui avait appris à attendre.

Par l'exercice de l'écriture récursive et l'échange en communauté de pairs, cet enseignant a vécu une transformation, une prise de conscience qui le met dans une attitude d'ouverture vers l'autre, nous dirons même en attente de l'autre. En même temps, nous pouvons voir qu'une telle transformation est propice à un changement chez l'élève qui finalement, transforme sa propre position envers l'apprentissage. Ainsi, cette pratique de professionnalisation va au de-là de la rhétorique, car la prise de conscience a pu transformer l'enseignement en véritable praxis.

\section{Discussion et retour sur la problématique}

\subsection{Convergence des processus de professionnalisation}

L'étude montre des possibilités réelles de construire entre les générations, dans le même espace-temps et sur la base d'une co-réflexivité, des éléments de l'identité et de l'éthique professionnelle. Cette édification est fortement favorisée si elle est ancrée dans une pratique d'écriture et d'analyse de cas réels. Il y a une richesse dans la convergence, facilitée par la reconnaissance mutuelle de l'expérience des enseignants et les connaissances qu'elle contient, ainsi que la vigueur des jeunes et des nouvelles idées des stagiaires.

Questions Vives, N² 24 | 2015 


$$
\begin{aligned}
& \text { séquence traditionnelle des parcours de formation, sans la nier entièrement car il y a des } \\
& \text { logiques qui sont propres à chaque étape de formation. La convergence donne des pistes } \\
& \text { pour résoudre les désaccords entre la théorie et la pratique et surtout, résout le problème } \\
& \text { de la valorisation et du respect professionnel mutuel. La convergence des itinéraires de } \\
& \text { formation permet l'appropriation d'une compréhension phénoménologique du champ } \\
& \text { d'action des enseignants, compréhension construite en communauté par l'ensemble des } \\
& \text { acteurs. Il s'ensuit des conséquences telles que des transitions vers de nouvelles } \\
& \text { dispositions d'action chez les enseignants ainsi que la restitution des liens dialogiques } \\
& \text { entre théorie et pratique, favorisant par-là même l'instauration d'une notion de la } \\
& \text { professionnalité construite tout au long de la vie. }
\end{aligned}
$$
professionnalité, nous avons observé que ce dispositif rompt initialement, avec la

\subsection{Développement professionnel des enseignants expérimentés et des stagiaires}

Du point de vue de la formation, le projet du dispositif des ateliers d'écriture a permis de redonner du pouvoir aux enseignants expérimentés, afin de prendre en charge leur professionnalisation, tout en favorisant chez eux l'examen de leur pratique professionnelle par une action réflexive, étayée par le "regard neuf» des jeunes stagiaires. Par ailleurs, ce projet a généré une conscience professionnelle à travers l'activité dialogique interdisciplinaire et intergénérationnelle au sein d'une communauté de pratique et d'écriture. Enfin, ce travail a pu conduire à des changements dans les propres actions des enseignants expérimentés avec un rôle plus actif vis-à-vis des modèles culturels de leurs institutions.

En ce qui concerne les futurs enseignants, ce projet semble avoir favorisé plus tôt, l'émergence des caractères de la profession tels que la valorisation et la systématisation par l'écrit de l'expérience d'enseignement, la capacité d'un travail réflexif en collaboration avec les pairs, le développement de nouvelles dispositions pédagogiques et d'enquête, la capacité de donner des réponses éthiques à des problèmes spécifiques de la pratique enseignante. Cet ensemble d'éléments participe à un développement de la conscience professionnelle et plus largement à la professionnalité.

\subsection{Enjeux/perspectives de formation}

Sur la scène latino-américaine de la formation des enseignants, il est difficile de trouver de façon explicite, des politiques intégrées de formation initiale et du développement professionnel des enseignants. Denise Vaillant (2007), coordonnatrice du Groupe de Travail sur le Développement Professionnel des Enseignants du PREAL ${ }^{7}$, met en relief ce point et parle d'un " chaînon manquant ». Celui-ci devrait être compris comme l'absence des dispositifs de soutien pour ceux qui débutent dans la carrière professionnelle. Nous 
estimons que ce « chaînon manquant » devrait prendre des formes de dispositifs réflexifs, servants aussi bien à soutenir les professeurs débutants que les expérimentés, tous engagés sur un chemin de professionnalisation: celui de la reconstruction et la transformation des enseignants.

Nous croyons que le rôle de l'enseignant tuteur est crucial dans ce processus d'introduction du stagiaire dans l'univers de l'école et dans la construction de l'identité professionnelle des futurs enseignants. Lors des stages, les tuteurs ont pour fonction d'aider à développer un regard pédagogique chez le futur enseignant. Le rôle de l'enseignant-tuteur serait de contribuer à construire une représentation de l'école et des classes.

Or, les enseignants tuteurs ne peuvent mettre en œuvre cette approche s'ils n'ont pas travaillé auparavant à la construction de leur propre regard. Certes, les enseignants ont développé un regard critique sur l'école et sur leurs pratiques tout au long de leur vie professionnelle, mais la plupart du temps, ce regard est resté non-dit, non explicité et, en conséquence, n'arrive pas à s'exprimer sous la forme de connaissances formalisées et transmissibles. Cette explicitation renvoie à des enjeux de formation. La construction de l'identité professionnelle est un processus de toute une vie, lié au développement de la conscience et favorisé par le langage. C'est la parole et son pouvoir de re-création des réalités qui permet à l'identité du professionnel de prendre forme.

Nous nous intéressons à l'écriture récursive en communauté de pratique, en tant que dispositif de recherche et de formation, visant au développement de la conscience professionnelle. La situation réflexive (Desgagné, 2005) choisie pour mettre en œuvre un tel objectif a été celle d'un atelier d'écriture de cas, situé en contexte scolaire réel. Nous avons vu alors, s'installer un principe d'herméneutique collective, qui favorise le dialogue de perspectives diverses chez les enseignants participant à l'expérience décrite ci-dessus, tout en visant l'émergence de caractéristiques propres qui commencent à être comprise par les membres de la communauté comme une identité en devenir (Malet, 1998). Cette idée est au centre de la transformation vécue par les enseignants engagés dans cette pratique réflexive. Si l'identité n'est pas fixe, si elle change, si le regard et les réponses éthiques aux problèmes vécus par les enseignants sont en développement et si l'apprentissage expérientiel s'installe comme un principe de professionnalité, il y a lieu de faire travailler ensemble, dans un même espace narratif, des enseignants qui ont dix, vingt ou trente ans de pratique, avec ceux qui commencent leur chemin dans la profession, avec un même objectif de professionnalisation.

C'est le principe du discernement qui vient prendre corps par ce travail entre pairs, entre ce qui est proprement pédagogique et ce qui ne l'est pas, entre diverses voies d'action. Il s'agit donc, pour la plupart des enseignants, d'une appréciation de nature éthique. C'est sur ce point que nous commençons à ouvrir le regard et à développer une compréhension épistémique de ce que représente l'objet du travail du professeur: le sujet en relation, apprenant et/ou enseignant, et celui-ci dans un contexte institutionnel largement reconnu comme étant en crise. Dans l'éveil d'une telle compréhension, l'écriture peutêtre une clé. 


\section{BIBLIOGRAPHIE}

Bakhtine [Bajtin], M. M. (1995). Estética de la creación verbal. Trad. Tatiana Bubnova, México: Siglo XXI.

Calderhead, J. (1996). Teachers: Beliefs and Knowledge. In Berliner \& Calfee (Eds.), Handbook of Educational Psychology (pp. 709-725). New York: Macmillan.

Castro Kikuchi, L. (2001) Diccionario de ciencias de la educación. Ediciones El Ecuador.

Clot, Y., \& Kostulski, K. (2011). Intervening for transforming: The horizon of action in the Clinic of Activity. Theory Psychology, 21(5), 681-696. En ligne http://tap.sagepub.com/content/21/5/681, DOI: $10.1177 / 0959354311419253$

Connelly, F. M., \& Clandinin, D.J. (1994). Telling teaching stories. Teacher Education Quarterly, 21(1), 145-158.

Desgagné, S. (1997). Le concept de recherche collaborative : l'idée d'un rapprochement entre chercheurs universitaires et praticiens enseignants. In Revue des sciences de l'éducation, 23(2), 371-393.

Desgagné, S., Bednarz, N., Couture, C., Poirier, L., \& Lebuis, P. (2001). L'approche collaborative de recherche en éducation : un rapport nouveau à établir entre recherche et formation. Revue des sciences de l'éducation, 27(1), En ligne http://www.erudit.org/revue/rse/2001/v27/

n1/000305ar.html

Desgagné, S. (2005). Récits exemplaires de pratique enseignante. Analyse typologique. Collection Éducation-Recherche. Québec : Presses de l'Université du Québec.

Elbaz, F. (1991). Research on Teacher's Knowledge: The Evolution of a Discourse. Journal of Curriculum Studies, 23(1), 1-19.

Freire, P. (2002). Cartas a quien pretende enseñar. Buenos Aires : Siglo XX

Giddens, A. (1987). La constitution de la société. Éléments de la théorie de la structuration.Paris : PUF.

Kleinfeld, J. (1992). Learning to Think Like a Teacher: The Study of Cases. In J.H. Shulman (éd.), Case Methods in Teacher Education (pp. 33-49).

Lieberman, A., \& Wood, D. (2006). Cuando los profesores escriben : sobre redes y aprendizajes. En Lieberman \& Miller, La Indagación como base de la formación del profesorado y la mejora de la educación (pp. 209-224). Barcelona : Editorial Octaedro.

Laforce, M. (2000). Des lilas à ciel ouvert. Québec. Le Loup de Gouttière.

Malet, R. (1998). L’identité en formation. Phénoménologie du devenir enseignant. Paris. Editions L'Harmattan.

Miller, Errol (2002). Políticas de formación docente en la mancomunidad del Caribe. En Formación docente : un aporte a la discusión. La experiencia de algunos países.UNESCO/OREALC, Santiago, Chile, 2002 (pp. 15-34).

Nieto, S. (2005). Why We Teach. New York: Teachers College Press. 
Núñez, M., Arévalo, A. \& Ávalos, B. (2012). Profesionalización docente : ¿Es posible un camino de convergencia para expertos y novatos ? Revista Electrónica de Investigación Educativa 14(2), 10-24. En ligne http://redie.uabc.mx/vol14no2/contenido-nunezetal.html

Schön, D. (1992). La formación de Profesionales Reflexivos : hacia un nuevo diseño de la enseñanza y el aprendizaje en las profesiones. Barcelona: Paidós

Shulman, L.S. (1992). Toward a Pedagogy of Cases. In J.H. Shulman, Case Methods in Teacher Education (pp. 1-30).

Shulman, L. (2005). Conocimiento y enseñanza : Fundamentos de la nueva reforma.Profesorado. Revista de currículum y formación del profesorado, vol. 9, ํㅜ 2, 1-30.

Torres, R. (2002). Prefacio. En P. Freire, Cartas a quien pretende enseñar (pp. 15-16). Buenos Aires : Siglo XXI Editores

Vaillant, D. (2007). Mejorando la formación y el desarrollo profesional docente en Latinoamérica. Pensamiento Educativo, vol. 41, № 2, 207-222

Wittorski, R. (2007). Professionnalisation et développement professionnel. Paris, L'Harmattan.

\section{NOTES}

1. El analfabetismo funcional es la situación del individuo que sabiendo leer y escribir no ejercita estas adquisiciones y, en los hechos, funciona como analfabeto por no utilizar lo que sabe (Castro, 2001, p. 29).

2. Première université chilienne du système public, fondée en 1842 .

3. Aujourd'hui en 2015, lesdites conditions sont objet des modifications comme résultats des mouvements citoyen vécus depuis 2011 au Chili et d'une réforme qui est encore en discussion parlementaire.

4. Clot (Clot \& Kostulski, 2011) identifie ce niveau de réflexivité comme étant le résultat d'une procédure d'autoconfrontation croisée. Il est fort intéressant de constater comment une même référence, ici Bakthine nous amène à un même point dans la réflexion ainsi qu'à la création d'un dispositif pour l'analyse de la pratique.

5. Type de conscience qui, pour nous, est développé sur les apprentissages et la manière dont ils ont été acquis. Résultat de la métacognition, uniquement l'activation d'une conscience épistémique permettrait de produire une base de connaissances professionnelles. Ce type de conscience, qui se manifeste par la voie de la curiosité et qui est favorisée par l'explicitation verbale, tant à l'oral qu'à l'écrit, a un impact réel dans les processus de professionnalisation, c'est-à-dire, dans la construction de l'identité, des dispositions éthiques et dans l'habileté à apprendre de l'expérience. C'est le niveau le plus développé par rapport à la conscience professionnelle.

6. Les stagiaires qui ont participé à ce projet font partie d'un programme de formation en enseignement secondaire qui exige comme condition d'être déjà titulaire d'un diplôme du baccalauréat. La durée du programme est de trois semestres, complémentaires à la formation disciplinaire de base.

7. PREAL, Programa de Promoción de la Reforma Educativa en América Latina y el Caribe / Partnership for Educational Revitalization in the Americas. http://www.preal.org/ENGL/ Default.asp. 


\section{RÉSUMÉS}

Le défi de la professionnalisation des professeurs se situe principalement au niveau du temps puisqu'il n'est réellement efficace que dans un processus de construction tout au long de la vie. Au Chili, comme ailleurs, elle est organisée en deux itinéraires de formation : initiale et continue. Nous croyons que, au-delà des divergences entre ces deux itinéraires, nous pourrions ouvrir intentionnellement une voie de convergence entre eux, afin de nourrir l'expérience des enseignants, qu'ils soient novices ou expérimentés. Nous proposons ici, une recherche collaborative en cours médiatisée par une situation réflexive : un atelier d'écriture de cas. Par cet atelier, un groupe constitué par des étudiants stagiaires et leurs tuteurs de stage, forme une communauté paritaire de pratique, aussi bien que d'enquête et d'écriture, par laquelle l'expérience professionnelle, individuelle et collective, est co-construite. Ainsi, l'idée de rapprocher les itinéraires de formation de ceux qui sont en début de carrière avec ceux qui ont plusieurs années d'expérience dans l'enseignement révèle tout un potentiel de transformation pour une professionnalisation plus aboutie.

Professionalization among teachers is a lifetime construction. However, it is generally organized in two separate learning pathways: initial and continuing training. We believe that, beyond the differences between these two paths, we could intentionally open a point of convergence between them, in order to mutually nurture the experience of novice and experienced teachers. We propose a collaborative research, supported by reflective action: a case writing workshop. Through this action, a group of teachers constituted by preservice teachers and their mentors begin to build a paritarian community of practice, investigation and writing. Thus, individual and collective professional experience will be co-constructed. The idea of bringing toghether the training routes of those who are at the begining of their career and those who have several years of teaching practice (in order to produce an intergenerational dialogue that would help to question, problematize and enrich the experience of novices and experienced teachers), is revealed in all its power of transformation as a professionalization device.

\section{INDEX}

Mots-clés : professionnalité, écriture de cas, conscience professionnelle, communautés de pratique

Keywords : professionalism, case writing, professional awareness, communities of practice

\section{AUTEUR}

\section{MAURICIO NÚÑEZ ROJAS}

Professeur adjoint au Département d'Études Pédagogiques à l'Université du Chili (Universidad de Chile) 